Applied nutritional investigation

\title{
Ketone bodies as a predictor of prognosis of hepatocellular carcinoma after transcatheter arterial chemoembolization
}

\author{
Ryu Sasaki M.D. a,b,*, Naota Taura M.D., Ph.D. b, Yuri Miyazoe M.D. ' , Shinobu Yamamichi M.D. ' \\ Suguru Nakashiki M.D. ', Mio Yamashima M.D. ' , Tomoyuki Suehiro M.D. b, Takuya Honda M.D., \\ Ph.D. ${ }^{\text {a,b }}$, Hidetaka Shibata M.D., Ph.D. ' , Eisuke Ozawa M.D., Ph.D. ' , Satoshi Miuma M.D., Ph.D. b, \\ Yuko Akazawa M.D., Ph.D. ', Hisamitsu Miyaaki M.D., Ph.D. b, Takehiro Matsumoto M.D., Ph.D. ', \\ Kazuhiko Nakao M.D., Ph.D. ${ }^{\text {b }}$ Kazuto Ashizawa M.D., Ph.D. ${ }^{\text {a }}$
}

a Department of Clinical Oncology, Unit of Translational Medicine, Nagasaki University Graduate School of Biomedical Sciences, Nagasaki City, Japan

${ }^{\mathrm{b}}$ Department of Gastroenterology and Hepatology, Nagasaki University Graduate School of Biomedical Sciences, Nagasaki City, Japan

\section{A R T I C L E I N F O}

\section{Article history:}

Received 23 October 2017

Received in revised form

11 December 2017

Accepted 29 December 2017

\section{Keywords:}

Intramuscular adipose tissue content

Skeletal muscle quality

Liver disease

Cirrhosis

Cancer

\section{A B S T R A C T}

Objective: Arterial ketone bodies, which reflect liver function, have been investigated. However, the relationship between venous ketone bodies and hepatocellular carcinoma (HCC) is unclear. We investigated whether prognosis of patients with HCC after transcatheter arterial chemoembolization (TACE) was associated with venous blood ketone bodies.

Methods: Sixty-eight patients with HCC who underwent TACE were recruited for this study. The venous blood ketone body levels were measured $1 \mathrm{~d}$ before (pretreatment) and $7 \mathrm{~d}$ after TACE (posttreatment). Skeletal muscle quality was evaluated using the intramuscular adipose tissue content (IMAC).

Results: Of the 68 patients, 43 (63.2\%) were male, with median age of $73.0 \mathrm{y}$, and the IMAC was -0.274 (range -0.82 to 0.24 ). The median ketone body levels pre- and posttreatment were $63.0 \mu \mathrm{mol} / \mathrm{L}$ (13$310)$ and $48.0 \mu \mathrm{mol} / \mathrm{L}(8-896)$, respectively. The cumulative survival rate of patients with total ketone body ratio ([TKBR]: posttreatment/pretreatment total ketone bodies) $<1$ was $86.6 \%$. The rate with TKBR $\geq 1$ was $59.0 \%$ at $300 \mathrm{~d}(P<0.05)$. Cox regression analysis identified the TKBR $(\geq 1$, hazard ratio: $2.954,95 \%$ confidence interval $[\mathrm{CI}]: 1.040-8.390, P=0.030)$ that independently and significantly predicted the patients' prognoses. Logistic regression analysis revealed the IMAC $(>-0.2745$, odds ratio: $3.958,95 \% \mathrm{CI}$ : 1.137-13.779, $P=0.031$ ) that predicted TKBR. TKBR and IMAC were positively correlated ( $\mathrm{rS}=0.358$, $P=0.003)$.

Conclusions: The changes in the venous ketone body were associated with the muscle status and predicted the prognosis of patients with HCC who underwent TACE. The venous ketone bodies could be a new predictor of the prognosis of HCC patients after TACE.

(C) 2018 Elsevier Inc. All rights reserved.

\section{Introduction}

Hepatocellular carcinoma (HCC) is the third most common cause of cancer-related deaths globally [1,2]. Patients with HCC are at an increased risk of being malnourished, and most HCCs develop as a result of chronic liver diseases and cirrhosis. Malnutrition is a common finding in patients with cancer and cirrhosis [3,4]. Moreover, sarcopenia is the major component of

The authors have no conflicts of interest to declare.

* Corresponding author. Tel.: +81958 19 7481; fax: +81958197482.

E-mail address: Ryu03202501@yahoo.co.jp (R. Sasaki). malnutrition, and it is a common complication in patients with chronic liver disease or cirrhosis [4-6]. Nutritional assessment of patients with chronic liver disease or cirrhosis is essential, because malnutrition is an independent predictor of mortality and complications [7-11]. However, there is no simple marker to assess nutrition or sarcopenia in HCC patients with chronic liver disease or cirrhosis.

Ketone bodies are composed of three molecules, 3-hydroxybutyrate (3-OHB), acetoacetate (AcAc), and acetone, which are produced from fatty acids in the liver. Ketone bodies play an important role in survival during starvation and provide a source of energy to the tissues in the brain, heart, muscle, and kidney in patients with glucose insufficiency [12]. 
Previous studies have reported on the relationship between hepatic reserve function and arterial ketone bodies during liver surgery or transcatheter arterial chemoembolization (TACE) [13-17]. However, no studies have focused on venous ketone bodies, nutrition status, and skeletal muscle status in patients with chronic liver disease, cirrhosis, or HCC. In this report, we used venous ketone bodies to assess the nutrition and skeletal muscle status, as well as predict the prognosis of patients with HCC who underwent TACE.

\section{Methods}

Patients

Between June 2014 and May 2015, 133 consecutive HCC patients who underwent TACE at our institution were enrolled in this retrospective study. HCC was diagnosed based on the positive results of typical vascular patterns, revealed by either contrast-enhanced computed tomography (CT), contrastenhanced magnetic resonance imaging, or angiography. Otherwise, the pathologic diagnosis was made via a fine-needle biopsy of space-occupying lesions that were detected in the liver.

The exclusion criteria for this study were 1 ) a shorter follow-up period $(<2 \mathrm{mo})$ after TACE treatment, 2) absence of properly examined samples or insufficient archival material, and 3) no definite HCC diagnosis. After the exclusion criteria were applied, data on 68 patients who underwent TACE were analyzed retrospectively.

\section{Measurement of blood samples and ketone bodies}

For all patients in our cohort, a blood sample was collected $1 \mathrm{~d}$ before (pretreatment) and $7 \mathrm{~d}$ after (posttreatment) TACE treatment. Medical histories, along with the results of routine tests for blood cell counts, liver biochemistry, and tumor markers at the time of TACE and thereafter were retrieved from the patients' medical records. Complete blood cell counts were obtained and biochemical tests were performed using automated procedures in the clinical pathology laboratories of our hospital. All blood samples were collected with the patients in a fasting state. The total ketone body level, 3-OHB, and AcAc were measured via an enzyme cycling method using a commercial kit (TKB-L Shiyaku Kainos, 3 HB-L Shiyaku Kainos; Kainos, Tokyo, Japan). We also calculated the total ketone body ratio (TKBR) by dividing the total ketone bodies at posttreatment (day 7 ) by the number of total ketone bodies at pretreatment (day 0 ).

Image analysis

The quality of the skeletal muscle was evaluated using the psoas muscle mass index (PMI) and intramuscular adipose tissue content (IMAC) using CT imaging. The cross-sectional areas of the right and left psoas muscles were measured with manual tracing on CT imaging at the $\mathrm{L} 3$ level to determine the PMI. The PMI was calculated by normalizing the cross-sectional areas for height $\left(\mathrm{cm}^{2} / \mathrm{m}^{2}\right)[18]$. The IMAC was determined by measuring the CT value of the multifidus muscles (Hounsfield units) and the $\mathrm{CT}$ value of subcutaneous fat (Hounsfield units) at the umbilical level. The IMAC was calculated by dividing the region of interest of the multifidus muscle (Hounsfield units) with the region of interest of the subcutaneous fat (Hounsfield units) [19,20].

\section{TACE treatment}

Among the 68 patients, 52 underwent conventional TACE (c-TACE) and 16 underwent drug-eluting bead (DEB)-TACE. The standard TACE procedure was performed via a right femoral artery puncture. Selective arteriography of the celiac and superior mesenteric arteries was performed to investigate the arterial anatomy of the liver, vascular supply of the tumor nodes, and patency of the portal vein. TACE was performed as selectively as possible. A mixture of metal matrix composite (Mitomycin-C; Kyowa, Tokyo, Japan) or epirubicin (Nippon Kayaku, Tokyo, Japan) that was manually emulsified with iodized oil (Lipiodol; Fuji Pharma Co., Tokyo, Japan) was used for the c-TACE procedures. The dosages of the chemotherapeutic drug used were determined according to the patient's body surface area and tumor size. Subsequently, embolization was performed with absorbable gelatin sponge particles (Gelpart; Nippon Kayaku, Tokyo, Japan) to reduce residual blood flow. DEB (DC Bead; Eisai Co., Tokyo, Japan) with a diameter of 100 to 300,300 to 500 , and 500 to $700 \mu \mathrm{m}$ was used for the DEB-TACE procedures. The dosage of epirubicin was $50 \mathrm{mg}$, which was equivalent to one vial of drug-eluting beads. Interventional radiologists clinically decided whether c-TACE or DEB-TACE should be performed.
Follow-up and diagnosis of HCC

All patients were followed up at an interval of 1 to 3 mo, the blood count and liver biochemistry were measured, and the $\alpha$-fetoprotein and des- $\gamma$ carboxy prothrombin levels were quantitatively detected. Diagnostic imaging either by ultrasound, CT, or magnetic resonance imaging was performed at least once every 3 mo.

\section{Ethical considerations}

Informed consent to use medical records and specimens was obtained from each patient. These processes and the study protocol were approved by the Ethical Committee of our institution (confirmation number: 16031421), and conformed to the 1975 Declaration of Helsinki and the Japanese Ethical Guidelines for Clinical Research (Ministry of Health, Labour, and Welfare of Japan, Ethical Guidelines for Clinical Research, 2008).

\section{Statistical analysis}

Continuous variables (albumin, prothrombin time, total bilirubin, $\alpha$-fetoprotein, fibrosis-4 [Fib-4], PMI, IMAC, and tumor size) were dichotomized with respect to the median value or clinically meaningful values in a multivariate analysis. A statistical analysis was performed using Wilcoxon's signed rank test and MannWhitney's $U$ test. To estimate the survival rate after TACE, we used the KaplanMeier method and the log-rank test. To select the optimal cut-off values of TKBR that indicate a poor prognosis after TACE, the area under the time-dependent receiver operating characteristic curves [21] was assessed. A Cox proportional hazards regression analysis was performed to evaluate the risk factors for survival after TACE. A multiple regression analysis was performed to determine the factors that were associated with TKBR. Age, sex, and variables with $P$ values $<0.20$ were selected and entered into the multiple regression model. A $P$ value of 0.05 was considered statistically significant. The data analysis was performed with SPSS Version 22.0 (IBM Corp., Armonk, NY, USA).

\section{Results}

\section{Patient characteristics}

The baseline characteristics of the 68 patients who were included in this study are summarized in Table 1 . The median age was $73.0 \mathrm{y}$; 43 patients (63.2\%) were male; branched chain amino acids were administered to 30 (44.1\%); and 3 (4.4\%), 24 (35.3\%), 26 (38.2\%), 14 (20.6\%), and 1 (1.5\%) patients were diagnosed with

Table 1

Characteristics of the patients enrolled in the present study

\begin{tabular}{ll}
\hline Factor & $n$ (range, \%) \\
\hline Age, y & $73.0(53-86)$ \\
Sex, male & $43(63.2 \%)$ \\
Period, d & $272.5(67-595)$ \\
BCAA, + & $30(44.1 \%)$ \\
BCLC stage 0/A/B/C/D & $3 / 24 / 26 / 14 / 1$ \\
Albumin, g/dL & $3.30(2.0-4.3)$ \\
PT-INR & $1.110(0.95-2.06)$ \\
Total bilirubin, mg/dL & $0.80(0.3-2.2)$ \\
Child-Pugh grade A/B/C & $44 / 23 / 1$ \\
AFP, ng/mL & $23.45(1.6-20182.0)$ \\
Fib-4 index & $5.98(1.2-14.0)$ \\
PMI, cm ${ }^{2} / m^{2}$ & $5.7005(2.392-8.729)$ \\
IMAC ratio & $-0.2745(-0.827$ to 0.239) \\
Tumor size, cm & $2.20(1.0-15.0)$ \\
Tumor number, single & $15(22.1 \%)$ \\
Etiology B/C/NBNC/alcohol & $10 / 34 / 13 / 11$ \\
DM & $21(30.9 \%)$ \\
TACE (conventional/DEB) & $52 / 16$
\end{tabular}

AFP, $\alpha$-fetoprotein; BCAA, branched chain amino acids; BCLC stage, Barcelona Clinic liver cancer stage; DEB, drug-eluting beads; DM, diabetes mellitus; Fib, fibrosis; IMAC, intramuscular adipose tissue content; INR, international normalized ratio; NBNC, non-B, non-C; PMI, psoas muscle mass index; PT, prothrombin time; TACE, transcatheter arterial chemoembolization.

Data are given as medians with ranges. Data were collected at pretreatment. 


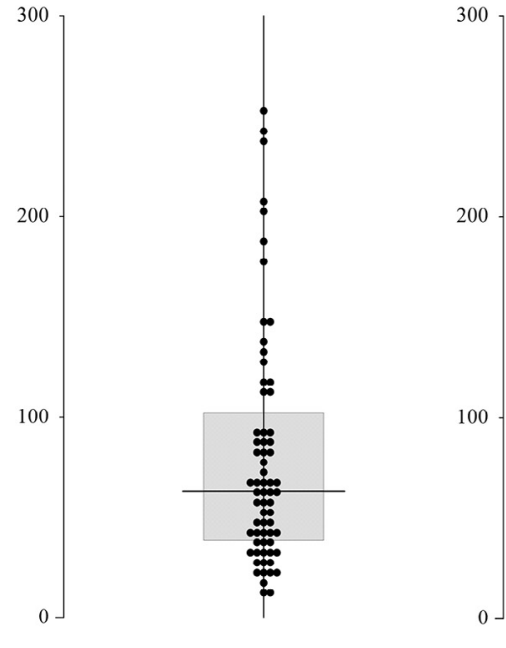

Total ketone bodies

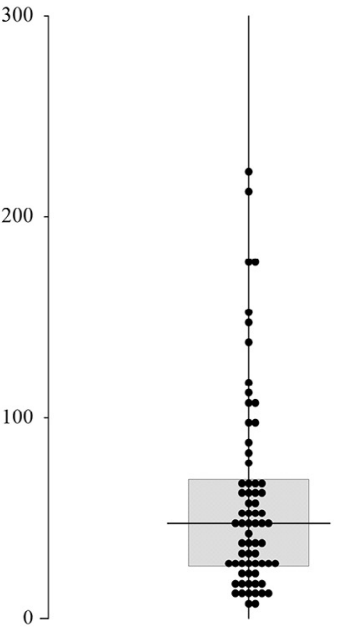

3-hydroxybutyrate
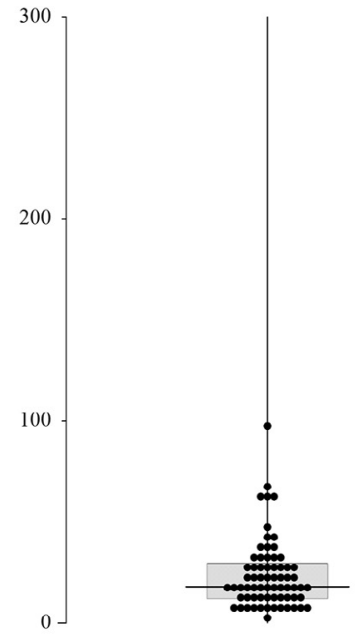

Acetoacetate

Fig. 1. Scatter plots of venous total ketone bodies, 3-hydroxybutyrate, and acetoacetate. The median values are indicated by the horizontal bars in the scatter plot. In the box plot, the bottom and top of the box are the 25th and 75th percentiles (the lower and upper quartiles), respectively.

Barcelona Clinic liver cancer (BCLC) stage 0, A, B, C, and D cancers, respectively. The median PMI was 5.70 (range: $2.39-8.72 \mathrm{~cm}^{2} / \mathrm{m}^{2}$ ), the IMAC was -0.274 (range: -0.82 to 0.24 ); the tumor size was 2.2 (range: $1.0-15.0 \mathrm{~cm}$ ), 15 patients $(22.1 \%$ ) were affected in a single nodule, and 21 patients (30.9\%) were diagnosed with diabetes mellitus. The median follow-up period was 272.5 (range: 67-595 d).

\section{Total ketone bodies at pretreatment}

The median total ketone body level, 3-OHB, and AcAc at pretreatment were 63.0 (range: $13-310 \mu \mathrm{mol} / \mathrm{L}$ ), 47.5 (range: 7-222 $\mu \mathrm{mol} / \mathrm{L}$ ), and 18.0 (range: $3-100 \mu \mathrm{mol} / \mathrm{L}$ ), respectively (Fig. 1). We divided the 68 patients into two groups stratified by the median value of the total ketone body level at pretreatment. In the group of patients whose total ketone body level was $\geq 63.0 \mu \mathrm{mol} / \mathrm{L}$, the following two factors were identified as significant in the univariate analysis: IMAC ratio and a history of diabetes mellitus (Table 2).

\section{Chronological changes in the total ketone body levels}

Figure 2 shows the changes in the total ketone body levels. The median value of the total ketone body level at pretreatment was 63.0 (range: $13-310 \mu \mathrm{mol} / \mathrm{L}$ ) and that of the total ketone body level at posttreatment was 48.0 (range 8-896 $\mu \mathrm{mol} / \mathrm{L}$ ). There was no significant change in the total ketone body level between pre- and posttreatment (Fig. 2A). However, in 40 patients (58.8\%), the total ketone body level after TACE decreased, and there was an increase in these levels in the other 28 patients (Fig. 2B). The median value of the pretreatment ketone body ratio (AcAc/3$\mathrm{OHB}$ ) was 0.41 (range: $0.1-1.3$ ) and that of posttreatment was

Table 2

Characteristics of the two groups, stratified by the median value of total ketone body level at pretreatment

\begin{tabular}{|c|c|c|c|}
\hline Factor & Total ketone bodies $<63 \mu \mathrm{mol} / \mathrm{L}(n=32)$ & Total ketone bodies $\geq 63 \mu \mathrm{mol} / \mathrm{L}(n=36)$ & $P$ \\
\hline Age, y & $73.5(60-86)$ & $73.0(53-85)$ & 0.60 \\
\hline Sex, male/female & $19 / 13$ & $24 / 12$ & 0.53 \\
\hline $\mathrm{BCAA}, \pm$ & $18 / 14$ & $20 / 16$ & 0.95 \\
\hline BCLC stage $0 / A / B / C / D$ & $2 / 12 / 12 / 5 / 1$ & $1 / 12 / 14 / 9 / 0$ & 0.45 \\
\hline Albumin, $\mathrm{g} / \mathrm{dL}$ & $3.30(2.0-4.2)$ & $3.30(2.6-4.3)$ & 0.63 \\
\hline PT-INR & $1.105(0.95-2.06)$ & $1.110(0.97-1.71)$ & 0.94 \\
\hline Total bilirubin, mg/dL & $0.80(0.4-2.0)$ & $0.90(0.3-2.2)$ & 0.27 \\
\hline Child-Pugh grade $\mathrm{A} / \mathrm{B} / \mathrm{C}$ & $17 / 14 / 1$ & $27 / 9 / 0$ & 0.12 \\
\hline AFP, $n g / m L$ & $13.00(1.9-16326.0)$ & $26.75(1.6-20182.0)$ & 0.27 \\
\hline Fib-4 index & $6.69(1.4-14.0)$ & $5.80(1.2-13.9)$ & 0.34 \\
\hline PMI, $\mathrm{cm}^{2} / \mathrm{m}^{2}$ & $5.2110(2.392-7.546)$ & $5.8340(3.285-8.729)$ & 0.07 \\
\hline IMAC ratio & $-0.2335(-0.53-0.239)$ & $-0.3425(-0.827$ to 0.056$)$ & $<0.01$ \\
\hline Tumor size, $\mathrm{cm}$ & $2.10(1.0-5.0)$ & $2.20(1.0-15.0)$ & 0.27 \\
\hline Tumor number, single/multiple & $9 / 23$ & $6 / 30$ & 0.25 \\
\hline Etiology HBV/HCV/non-B, non-C/alcohol & $2 / 20 / 6 / 4$ & $8 / 14 / 7 / 7$ & 0.84 \\
\hline $\mathrm{DM}, \pm$ & $26 / 6$ & $18 / 18$ & $<0.01$ \\
\hline TACE conventional/DEB & $24 / 8$ & $28 / 8$ & 0.78 \\
\hline
\end{tabular}

AFP, $\alpha$-fetoprotein; BCAA, branched chain amino acids; BCLC stage, Barcelona Clinic liver cancer stage; DEB, drug-eluting beads; DM, diabetes mellitus; Fib, fibrosis; HBV, hepatitis B virus; HCV, hepatitis C virus; IMAC, intramuscular adipose tissue content; INR, international normalized ratio; PMI, psoas muscle mass index; PT, prothrombin time; TACE, transcatheter arterial chemoembolization.

A $\chi^{2}$ and Mann-Whitney $U$ test were performed for comparisons.

Data are given as medians with ranges. Data were collected at pretreatment. 
a

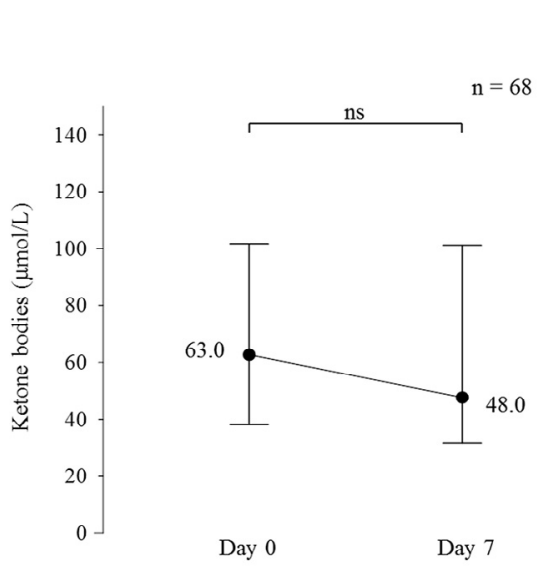

b

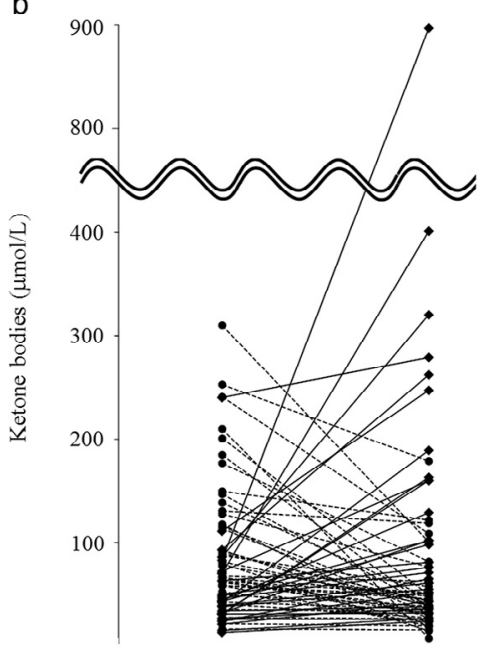

Day 0

Day 7

Fig. 2. Chronological changes in the total ketone body levels. Chronological changes in the total ketone body level at pretreatment (day 0) and posttreatment (day 7) of the 68 HCC patients who underwent TACE. (A) The total ketone bodies were not significantly changed after treatment. The dots represent the median serum total ketone body values at each time point, and the error bar represents the interquartile range. (B) The changes in the total ketone body level in individual patients. The solid line indicates the group of patients in whom the level increased $(n=28)$. The dashed line indicates those in whom the level decreased ( $n=40)$. Wilcoxon's signedrank test was performed for comparisons. HCC, hepatocellular carcinoma; TACE, transcatheter arterial chemoembolization.

0.45 (range: $0.2-2.3$ ). There was no significant change between the pre- and posttreatment ketone body ratios (data not shown).

\section{Cumulative survival rate after TACE}

During the follow-up period, 16 of the 68 patients (23.5\%) died. The cumulative survival rate was $97.1 \%$ at $100 \mathrm{~d}, 86.2 \%$ at $200 \mathrm{~d}$, and $76.6 \%$ at $300 \mathrm{~d}$. To evaluate the relationship between the total ketone body level and survival after TACE, we characterized these 68 patients according to their total ketone body level at pretreatment and TKBR (posttreatment/pretreatment total ketone bodies).
Figure $3 \mathrm{~A}$ shows the survival rate after TACE, stratified by the median value of the total ketone bodies at pretreatment. There were no significant differences in the survival rate between total ketone bodies $\geq 63.0 \mu \mathrm{mol} / \mathrm{L}$ group and $<63.0 \mu \mathrm{mol} / \mathrm{L}$ group at pretreatment $(P=0.61$ in the log-rank test). Figure 3B shows the survival rate after TACE, stratified by the TKBR. The 200-, 300-, and $400-d$ survival rates were $86.6 \%, 86.6 \%$, and $81.5 \%$, respectively, in the 40 patients with TKBR $<1$ (reduced group); and $86.0 \%$, $59.0 \%$, and $50.6 \%$, respectively, in the 28 patients with TKBR $\geq 1$ (raised group). The survival rates were significantly higher in the reduced group than in the raised group $(P<0.05$ in the logrank test).

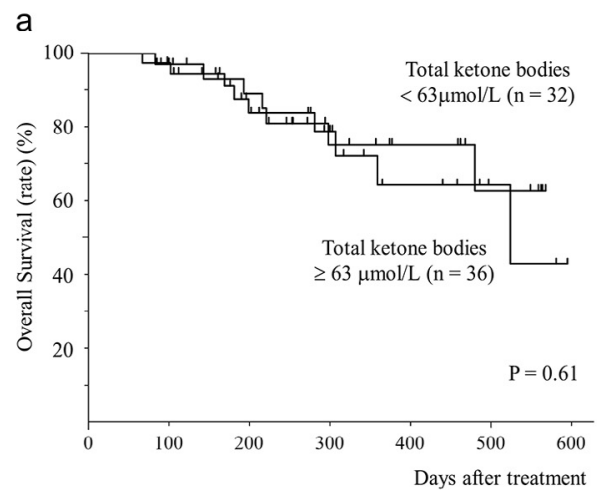

b

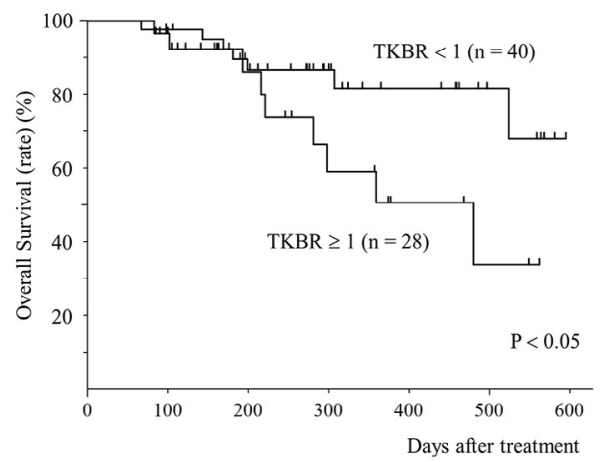

\begin{tabular}{ccccc} 
Overall Survival (rate) $(\%)$ & 100 -day & 200 -day & 300 -day & 400 -day \\
\hline $\begin{array}{c}\text { Total ketone bodies } \\
<63 \mu \mathrm{mol} / \mathrm{L}(\mathrm{n}=32)\end{array}$ & $96.9 \%$ & $88.9 \%$ & $75.1 \%$ & $75.1 \%$ \\
$\begin{array}{c}\text { Total ketone bodies } \\
\geq 63 \mu \mathrm{mol} / \mathrm{L}(\mathrm{n}=36)\end{array}$ & $97.2 \%$ & $83.6 \%$ & $78.7 \%$ & $64.1 \%$ \\
\hline
\end{tabular}

\begin{tabular}{ccccc} 
Overall Survival (rate) $(\%)$ & 100 -day & 200 -day & 300 -day & 400 -day \\
\hline TKBR $<1(\mathrm{n}=40)$ & $97.5 \%$ & $86.6 \%$ & $86.6 \%$ & $81.5 \%$ \\
TKBR $>1(\mathrm{n}=28)$ & $96.4 \%$ & $86.0 \%$ & $59.0 \%$ & $50.6 \%$ \\
\hline
\end{tabular}

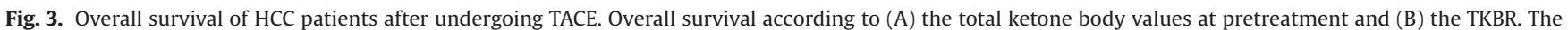

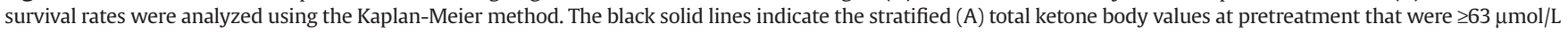

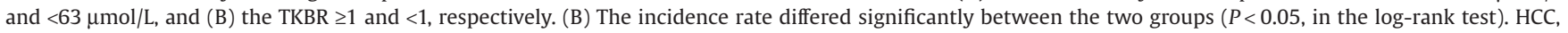
hepatocellular carcinoma; TACE, transcatheter arterial chemoembolization; TKBR, total ketone body ratio. 
Table 3

Risk factors associated with survival after TACE

\begin{tabular}{|c|c|c|c|c|c|}
\hline \multicolumn{3}{|l|}{ Univariate analysis } & \multicolumn{3}{|c|}{ Multivariate analysis } \\
\hline Risk factor & & $P$ & Hazard ratio & (95\% confidence interval) & $P$ \\
\hline Age, $y$ & $>73$ & 0.21 & & & \\
\hline Sex & Male & 0.74 & & & \\
\hline BCAA & + & 0.22 & & & \\
\hline BCLC stage & $\mathrm{B} / \mathrm{C} / \mathrm{D}$ & 0.35 & & & \\
\hline Albumin, g/dL & $>3.3$ & 0.81 & & & \\
\hline PT-INR & $<1.110$ & 0.38 & & & \\
\hline Total bilirubin, mg/dL & $>0.8$ & 0.65 & & & \\
\hline Child-Pugh grade & $\mathrm{B} / \mathrm{C}$ & 0.92 & & & \\
\hline AFP, ng/mL & $>23.45$ & 0.87 & & & \\
\hline Fib-4 index & $>6.0$ & 0.19 & 0.522 & $(0.183-1.485)$ & 0.223 \\
\hline PMI, $\mathrm{cm}^{2} / \mathrm{m}^{2}$ & $<5.70$ & 0.38 & & & \\
\hline IMAC ratio & $>-0.2745$ & 0.48 & & & \\
\hline Tumor size, $\mathrm{cm}$ & $>2.20$ & 0.11 & 1.272 & $(0.459-3.520)$ & 0.642 \\
\hline Tumor number & Multiple & 0.63 & & & \\
\hline DM & + & 0.77 & & & \\
\hline TACE & DEB & 0.33 & & & \\
\hline Total ketone bodies, $\mu \mathrm{mol} / \mathrm{L}$ & $\geq 63.0$ & 0.61 & & & \\
\hline TKBR & $\geq 1$ & $<0.01$ & 2.954 & $(1.040-8.390)$ & 0.03 \\
\hline
\end{tabular}

AFP, $\alpha$-fetoprotein; BCAA, branched chain amino acids; BCLC stage, Barcelona Clinic liver cancer stage; DEB, drug-eluting beads; DM, diabetes mellitus; Fib, fibrosis;

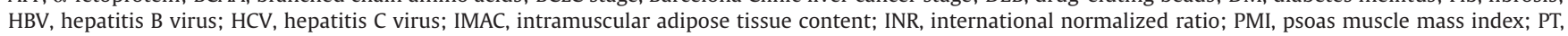
prothrombin time; TACE, transcatheter arterial chemoembolization; TKBR, total ketone body ratio.

Risk factors associated with survival after TACE Hazard ratios for the development of hepatocellular carcinoma were calculated using Cox proportional hazards analysis.

\section{Risk factors for survival after TACE}

The univariate analysis identified the factors that influence the risk for survival after TACE. A Cox regression analysis was performed for 18 variables: age, sex, branched chain amino acid levels, BCLC stage, albumin, prothrombin time, total bilirubin, ChildPugh grade, $\alpha$-fetoprotein, Fib-4 index, PMI, IMAC, tumor size, tumor number, diagnosis of diabetes mellitus, type of TACE, total ketone body level, and TKBR. The following three factors were identified as risk factors for survival after TACE using the univariate analysis $(P<0.20)$ : Fib-4 index, tumor size, and TKBR (Table 3).

A multivariate analysis was performed on the three factors (Fib-4 index, tumor size, and TKBR) identified via univariate analysis $(P<0.20)$. TKBR $(\geq 1$, hazard ratio: $2.954,95 \%$ confidence interval $[C I]: 1.040-8.390, P=0.030)$ was identified as an independent and significant risk factor of patient prognosis after TACE (Table 3).

\section{Predictors for TKBR}

We divided the patients into two groups, stratified by TKBR. The following nine significant $(P<0.20)$ factors were identified in the TKBR raised group in the univariate analysis: age, sex, BCLC stage, prothrombin time, total bilirubin, PMI, IMAC, tumor size, and diagnosis of diabetes mellitus. Multivariable logisticregression models were prepared to estimate the predictors for the TKBR. A multivariate logistic regression analysis revealed that the IMAC (>-0.2745, odds ratio: $3.958,95 \%$ CI: $1.137-13.779$, $P=0.031)$ and tumor size $(>2.2 \mathrm{~cm}$, odds ratio: $4.115,95 \% \mathrm{CI}$ : 1.072-15.796, $P=0.039$ ) were predictors for the TKBR (Table 4 ).

\section{Correlation between the TKBR and IMAC}

Figure 4 shows a scatter plot of the TKBR and the IMAC. The values that were obtained for the TKBR and IMAC groups in the Spearman rank order correlation test $(\mathrm{rS}=0.358)$ indicated that there was a positive correlation between the groups $(P=0.003)$.

\section{Discussion}

Ketone bodies are small lipid-derived molecules that are a source of energy for the peripheral tissues during fasting or prolonged exercise [22]. During fasting, the muscle and liver stores of glycogen are depleted first. Then, fatty acids are transported

Table 4

Predictors of the total ketone body ratio (total ketone bodies on day $7 /$ total ketone bodies on day 0 )

\begin{tabular}{|c|c|c|c|c|}
\hline \multirow[t]{2}{*}{ Independent variables } & & \multicolumn{3}{|c|}{ Multivariate model } \\
\hline & & Odds ratio & $\begin{array}{l}\text { (95\% confidence } \\
\text { interval) }\end{array}$ & $P$ \\
\hline \multirow[t]{2}{*}{ Age, y } & $\leq 73$ & 1 & (reference) & \\
\hline & $>73$ & 1.319 & $(0.416-4.182)$ & 0.638 \\
\hline \multirow[t]{2}{*}{ Sex } & Male & 1 & (reference) & \\
\hline & Female & 1.551 & $(0.376-6.398)$ & 0.544 \\
\hline \multirow[t]{2}{*}{ BCLC stage } & $0 / \mathrm{A}$ & 1 & (reference) & \\
\hline & $\mathrm{B} / \mathrm{C} / \mathrm{D}$ & 0.641 & $(0.162-2.543)$ & 0.527 \\
\hline \multirow[t]{2}{*}{ PT-INR } & $\leq 1.110$ & 1 & (reference) & \\
\hline & $>1.110$ & 0.682 & $(0.210-2.221)$ & 0.526 \\
\hline \multirow[t]{2}{*}{ Total bilirubin, mg/dL } & $\leq 0.8$ & 1 & (reference) & \\
\hline & $>0.8$ & 0.325 & $(0.096-1.101)$ & 0.071 \\
\hline \multirow[t]{2}{*}{ PMI, $\mathrm{cm}^{2} / \mathrm{m}^{2}$} & $\geq 5.70$ & 1 & (reference) & \\
\hline & $<5.70$ & 2.426 & $(0.651-9.035)$ & 0.187 \\
\hline \multirow[t]{2}{*}{ IMAC } & $\leq-0.2745$ & 1 & (reference) & \\
\hline & $>-0.2745$ & 3.958 & (1.137-13.779) & 0.031 \\
\hline \multirow[t]{2}{*}{ Tumor size, cm } & $\leq 2.2$ & 1 & (reference) & \\
\hline & $>2.2$ & 4.115 & $(1.072-15.796)$ & 0.039 \\
\hline \multirow[t]{2}{*}{ DM } & - & 1 & (reference) & \\
\hline & + & 0.823 & $(0.226-3.001)$ & 0.768 \\
\hline
\end{tabular}

BCLC stage, Barcelona Clinic liver cancer stage; DM, diabetes mellitus; IMAC, intramuscular adipose content; INR, international normalized ratio; PMI, psoas muscle mass index; PT, prothrombin time.

Multivariable logistic-regression models were used to estimate the predictors for the total ketone body ratio. Variables were included in the model based on the univariate analysis $(P<0.20)$. 


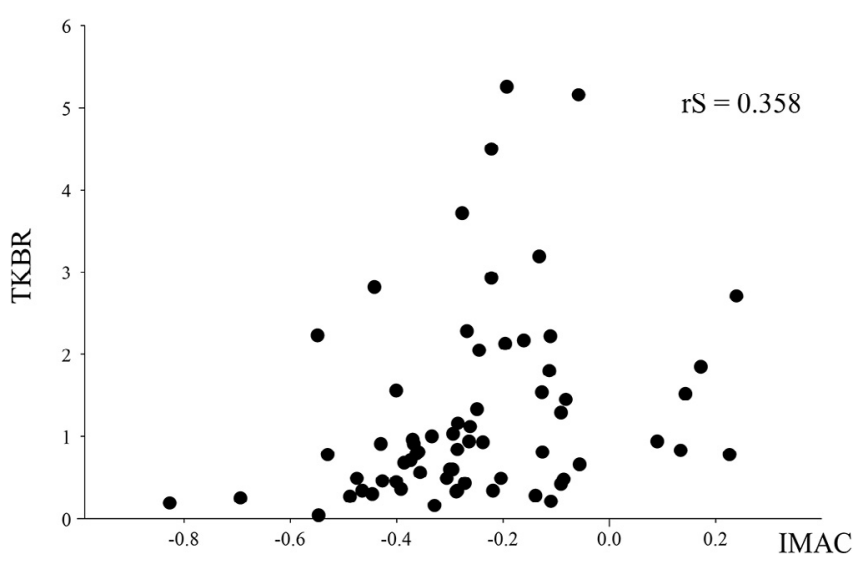

Fig. 4. Correlation between the TKBR and IMAC. Scatter plot of the TKBR and IMAC. The values that were obtained for the TKBR and IMAC groups using Spearman's rank order correlation test $(\mathrm{rS}=0.358$ ) indicated the presence of positive correlations between the groups ( $P=0.003)$. IMAC; intramuscular adipose content; TKBR, total ketone body ratio.

to the liver for conversion to ketone bodies. Patients with advanced liver disease particularly have increased fat oxidation [11]. The production of ketone bodies plays an important role in metabolites. Serum ketone bodies are defined by a variety of factors, such as energy metabolism, circumstances (starving, prolonged exercise, low-carbohydrate diets, and diabetes mellitus), liver conditions, and the extrahepatic tissues (heart, kidney, brain, and skeletal muscle). Previous studies have focused on arterial ketone bodies and liver function [14,16]. Conversely, the extrahepatic tissues are assumed to influence venous ketone bodies.

The first main finding of our study was that venous ketone bodies had different dynamics from those of arterial ketone bodies. Previous studies identified a relationship between the arterial ketone body ratio (AcAc/3-OHB) and hepatic reserve function $[15,16]$. However, there was no relationship between venous ketone bodies and hepatic reserve function in our study (Tables 2 and 4). Furthermore, there was no significant change in the venous ketone body ratio (AcAc/3-OHB) after TACE. Recent studies reported that ketone bodies regulate metabolism and 3-OHB signals via extracellular receptors and endogenously inhibit histone deacetylases [23]. Suppression of oxidative stress as a result of 3-OHB may benefit organs after a patient undergoes TACE. In our study, in more than half of the patients, the total ketone body level decreased after TACE. Thus, the elevation of ketone bodies $7 \mathrm{~d}$ after TACE does not solely reflect ischemic changes.

The second main finding of our study was that the TKBR can predict the patient's prognosis after undergoing TACE. We were able to stratify the patients into different risk groups using the TKBR (Fig. 3). The multivariate analysis revealed that the TKBR was the most significant factor for survival after TACE (Table 3). There was no correlation between the TKBR and hepatic functional reserve. The reason why TKBR predicts prognosis was unclear. However, our study revealed that elevation of the total ketone body level was related to an extrahepatic factor (skeletal muscle).

The third main finding of our study was that the quality of the skeletal muscle (IMAC) affected the ketone body level after TACE. The multivariate analysis revealed that the quality of the skeletal muscle (IMAC) was the significant factor that predicted the TKBR (Table 4). We found that the TKBR significantly correlated with the IMAC (Fig. 4). A high TKBR allowed us to identify patients with low muscle quality. Skeletal muscle depletion, which indicates a low quantity and quality of skeletal muscle, is referred to as sarcopenia and predicts mortality in patients with advanced liver disease [24-26]. Based on these considerations, we suggest that the TKBR does not reflect the hepatic reserve function but rather the nutritional status of patients with HCC who underwent TACE. Venous ketone bodies may be associated with nutritional status and sarcopenia in HCC patients.

The present study was limited by its retrospective nature. A future prospective analysis is needed to validate the efficacy of the total ketone body ratio to predict patients' prognoses after undergoing TACE. Another limitation is that there were no definite criteria to estimate the quantity and quality of the skeletal muscle. In addition, the ketone bodies were influenced by several factors (food intake, administration of drugs, and exercise). Other elements, which we did not evaluate, might have affected the ketone body level. Impaired performance status, advanced stages of disease, and poor hepatic reserve function were associated with shorter survival of patients with HCC [27]. In our study, the ChildPugh grade or BCLC stage was not a significant factor for survival in patients who underwent TACE. One plausible explanation is that our study consisted of patients with similar backgrounds regarding liver function and disease progression. Furthermore, the small number of participants in this study is also a limitation.

Regardless of these limitations, this is the first report to confirm the relationship between venous ketone bodies and treatment of HCC. In addition, the TKBR may predict the patient's prognosis and be related to the quality of skeletal muscle. Several studies reported that the quantity and quality of the skeletal muscle are important for achieving good clinical outcomes in patients with advanced liver diseases [5,24-26,28]. Increasing the skeletal muscle mass and function may be a possible therapeutic target to improve the prognosis of patients with advanced HCC. Therefore, the prediction of patients with poor prognosis after treatment is of increasing clinical relevance.

\section{Conclusion}

In conclusion, this study revealed that there was an association between venous ketone bodies and survival of HCC patients who underwent TACE. Furthermore, the venous ketone bodies in HCC patients who underwent TACE were useful to predict skeletal muscle quality. The results suggest that venous ketone bodies could be a new predictor of prognosis of HCC patients after TACE.

\section{References}

[1] Bruix J, Llovet JM. Major achievements in hepatocellular carcinoma. Lancet 2009;373:614-16

[2] El-Serag HB. Hepatocellular carcinoma: an epidemiologic view. J Clin Gastroenterol 2002;35:S72-8.

[3] Jensen GL, Bistrian B, Roubenoff R, Heimburger DC. Malnutrition syndromes: a conundrum vs continuum. JPEN J Parenter Enteral Nutr 2009;33:71016.

[4] Alberino F, Gatta A, Amodio P, Merkel C, Di Pascoli L, Boffo G, et al. Nutrition and survival in patients with liver cirrhosis. Nutrition 2001;17:44550.

[5] Dasarathy S, Merli M. Sarcopenia from mechanism to diagnosis and treatment in liver disease. J Hepatol 2016;65:1232-44.

[6] Merli M, Riggio O, Dally L. Does malnutrition affect survival in cirrhosis? PINC (Policentrica Italiana Nutrizione Cirrosi). Hepatology 1996;23:10416.

[7] Alvares-da-Silva MR, Reverbel da Silveira T. Comparison between handgrip strength, subjective global assessment, and prognostic nutritional index in assessing malnutrition and predicting clinical outcome in cirrhotic outpatients. Nutrition 2005;21:113-17.

[8] Cabre E, Gassull MA. Nutrition in liver disease. Curr Opin Clin Nutr Metab Care 2005;8:545-51.

[9] Norman K, Kirchner H, Lochs H, Pirlich M. Malnutrition affects quality of life in gastroenterology patients. World J Gastroenterol 2006;12:3380-5. 
[10] Cheung K, Lee SS, Raman M. Prevalence and mechanisms of malnutrition in patients with advanced liver disease, and nutrition management strategies. Clin Gastroenterol Hepatol 2012;10:117-25.

[11] Tajika M, Kato M, Mohri H, Miwa Y, Kato T, Ohnishi H, et al. Prognostic value of energy metabolism in patients with viral liver cirrhosis. Nutrition 2002; 18:229-34.

[12] Hegardt FG. Mitochondrial 3-hydroxy-3-methylglutaryl-CoA synthase: a control enzyme in ketogenesis. Biochem J 1999;338(Pt 3):569-82.

[13] Hanazaki K, Wakabayashi M, Sodeyama H, Makiuchi A, Igarashi J, Yokoyama $\mathrm{S}$, et al. Arterial ketone body ratio does not correlate with ischemic changes during major hepatectomy. Hepatogastroenterology 1998;45:1459.

[14] Matsushita K, Kawasaki S, Makuuchi M. Arterial ketone body ratio in liver surgery. Hepatology 1994;20:331-5.

[15] Tani T, Taki Y, Jikko A, Minematsu S, Yamamoto M, Kamiyama T. Shortterm changes in blood ketone body ratios in the phase immediately after hepatic artery embolization: their clinical significance. Am J Med Sci 1986; 291:93-100.

[16] Yamamoto R. Changes in arterial ketone body ratio after transcatheter arterial embolization for hepatocellular carcinoma-clinical and experimental studies. Nihon Shokakibyo Gakkai Zasshi 1990;87:1401-9.

[17] Yamaoka K, Kanayama M, Tajiri K, Yamane M, Marumo F, Sato C. Clinical significance of arterial ketone body ratio in chronic liver disease. Digestion 1998;59:360-3.

[18] Hamaguchi Y, Kaido T, Okumura S, Kobayashi A, Hammad A, Tamai Y, et al. Proposal for new diagnostic criteria for low skeletal muscle mass based on computed tomography imaging in Asian adults. Nutrition 2016;32:12005.
[19] Kitajima Y, Eguchi Y, Ishibashi E, Nakashita S, Aoki S, Toda S, et al. Agerelated fat deposition in multifidus muscle could be a marker for nonalcoholic fatty liver disease. J Gastroenterol 2010;45:218-24.

[20] Kitajima Y, Hyogo H, Sumida Y, Eguchi Y, Ono N, Kuwashiro T, et al. Severity of non-alcoholic steatohepatitis is associated with substitution of adipose tissue in skeletal muscle. J Gastroenterol Hepatol 2013;28:1507-14.

[21] Heagerty PJ, Lumley T, Pepe MS. Time-dependent ROC curves for censored survival data and a diagnostic marker. Biometrics 2000;56:337-44.

[22] Cahill GF. Fuel metabolism in starvation. Annu Rev Nutr 2006;26:1-22.

[23] Shimazu T, Hirschey MD, Newman J, He W, Shirakawa K, Le Moan N, et al. Suppression of oxidative stress by beta-hydroxybutyrate, an endogenous histone deacetylase inhibitor. Science 2013;339:211-14.

[24] Hamaguchi Y, Kaido T, Okumura S, Fujimoto Y, Ogawa K, Mori A, et al. Impact of quality as well as quantity of skeletal muscle on outcomes after liver transplantation. Liver Transpl 2014;20:1413-19.

[25] Harimoto N, Yoshizumi T, Shimokawa M, Sakata K, Kimura K, Itoh S, et al. Sarcopenia is a poor prognostic factor following hepatic resection in patients aged 70 years and older with hepatocellular carcinoma. Hepatol Res 2016;46:1247-55.

[26] Levolger S, van Vledder MG, Muslem R, Koek M, Niessen WJ, de Man RA, et al. Sarcopenia impairs survival in patients with potentially curable hepatocellular carcinoma. J Surg Oncol 2015;112:208-13.

[27] Cabibbo G, Enea M, Attanasio M, Bruix J, Craxi A, Camma C. A metaanalysis of survival rates of untreated patients in randomized clinical trials of hepatocellular carcinoma. Hepatology 2010;51:1274-83.

[28] Durand F, Buyse S, Francoz C, Laouenan C, Bruno O, Belghiti J, et al. Prognostic value of muscle atrophy in cirrhosis using psoas muscle thickness on computed tomography. J Hepatol 2014;60:1151-7. 